Please do not remove this page

RMIT

UNIVERSITY

\title{
Exfoliation of quasi-stratified Bi2S3 crystals into micron-scale ultrathin corrugated nanosheets
}

Clark, Rhiannon; Kotsakidis, Jimmy; Weber, Bent; Berean, Kyle; Carey, Benjamin; Field, Matthew; Khan, Hareem

https://researchrepository.rmit.edu.au/esploro/outputs/9921862101201341/filesAndLinks?institution=61RMIT_INST\&index=null

Clark, R., Kotsakidis, J., Weber, B., Berean, K., Carey, B., Field, M., Khan, H., Ou, J., Ahmed, T., Harrison, C., Cole, I., Latham, K., Kalantar Zadeh, K., \& Daeneke, T. (2016). Exfoliation of quasi-stratified Bi2S3 crystals into micron-scale ultrathin corrugated nanosheets. Chemistry of Materials, 28(24), 8942-8950.

https://doi.org/10.1021/acs.chemmater.6b03478

Document Version: Accepted Manuscript

Published Version: https://doi.org/10.1021/acs.chemmater.6b03478

Repository homepage: https://researchrepository.rmit.edu.au

(c) 2016 American Chemical Society

Downloaded On 2023/04/26 22:14:46 +1000 
Thank you for downloading this document from the RMIT Research Repository.

The RMIT Research Repository is an open access database showcasing the research outputs of RMIT University researchers.

RMIT Research Repository: http://researchbank.rmit.edu.aul

\author{
Citation: \\ Clark, R, Kotsakidis, J, Weber, B, Berean, K, Carey, B, Field, M, Khan, H, Ou, J, \\ Ahmed, T, Harrison, C, Cole, I, Latham, K, Kalantar Zadeh, K and Daeneke, T 2016, \\ 'Exfoliation of quasi-stratified Bi2S3 crystals into micron-scale ultrathin corrugated \\ nanosheets', Chemistry of Materials, vol. 28, no. 24, pp. 8942-8950.
}

See this record in the RMIT Research Repository at:

https://researchbank.rmit.edu.au/view/rmit:42466

Version: Accepted Manuscript

Copyright Statement:

(c) 2016 American Chemical Society

Link to Published Version:

http://dx.doi.org/10.1021/acs.chemmater.6b03478 


\title{
Exfoliation of quasi-stratified $\mathrm{Bi}_{2} \mathrm{~S}_{3}$ crystals into micron-scale ul- trathin corrugated nanosheets
}

\author{
Rhiannon M. Clark ${ }^{\mathrm{a}, \mathrm{b}}$, Jimmy C. Kotsakidis ${ }^{\mathrm{c}, \mathrm{d}}$, Bent Weber ${ }^{\mathrm{c}, \mathrm{d}}$, Kyle J. Berean ${ }^{\mathrm{a}}$, Benjamin J. Carey ${ }^{\mathrm{a}, \mathrm{b}}$, \\ Matthew R. Field ${ }^{\mathrm{e}, \mathrm{f}}$, Hareem Khan ${ }^{\mathrm{a}}$, Jian Zhen Ou ${ }^{\mathrm{a}}$, Taimur Ahmed ${ }^{\mathrm{g}}$, Christopher J. Harrison ${ }^{\mathrm{a}}$, \\ Ivan S. Cole ${ }^{\mathrm{b}}$, Kay Latham ${ }^{\mathrm{f}}$, Kourosh Kalantar-zadeh ${ }^{\mathrm{a} *}$, Torben Daeneke ${ }^{\mathrm{a} *}$. \\ ${ }^{a}$ School of Engineering, RMIT University, Melbourne, 3000, Australia, ${ }^{\mathrm{b}}$ Manufacturing, CSIRO, Clayton, 3168, Aus- \\ tralia, ${ }^{\mathrm{c}}$ School of Physics and Astronomy, Monash University, Clayton, 3800 , Australia, ${ }^{\mathrm{d}}$ Monash Centre for Atomi- \\ cally Thin Materials, Monash University, Clayton, 380o, Australia, ${ }^{\mathrm{e}}$ RMIT Microscopy and Microanalysis Facility, \\ RMIT University, Melbourne, 300o, Australia, ${ }^{\mathrm{f}}$ School of Science, RMIT University, Melbourne, 300o, Australia, \\ ${ }^{\mathrm{g}}$ Functional Materials and Microsystems Research Group, RMIT University, Melbourne, 300o, Australia
}

\begin{abstract}
There is ongoing interest in exploring new two-dimensional materials and exploiting their functionalities. Here, a top-down approach is used for developing a new morphology of ultrathin nanosheets from highly ordered bismuth sulfide crystals. The efficient chemical delamination method exfoliates the bulk powder into a suspension of corrugated ultrathin sheets, despite the fact that the $\mathrm{Bi}_{2} \mathrm{~S}_{3}$ fundamental layers are made of atomically thin ribbons that are held together by van der Waals forces in two dimensions. Morphological analyses show that the produced corrugated sheets are as thin as $2.5 \mathrm{~nm}$, and can be as large as $20 \mu \mathrm{m}$ across. Determined atomic ratios indicate that the exfoliation process introduces sulfur vacancies into the sheets, with a resulting stoichiometry of $\mathrm{Bi}_{2} \mathrm{~S}_{2.6}$. It is hypothesized that the nanoribbons were cross-linked during the reduction process leading to corrugated sheet formation. The material is used for preparing field effect devices and was found to be highly p-doped, which is attributed to the substoichiometry. These devices show a near-linear response to the elevation of temperature. The devices demonstrate selective and relatively fast response to $\mathrm{NO}_{2}$ gas when tested as gas sensors. This is the first report showing the possibility of exfoliating planar morphologies of metal chalcogenide compounds such as orthorhombic $\mathrm{Bi}_{2} \mathrm{~S}_{3}$, even if their stratified crystal structures constitute van der Waals forces within the fundamental planes.
\end{abstract}

\section{Introduction}

Among the group V-VI semiconductors, bismuth sulfide $\left(\mathrm{Bi}_{2} \mathrm{~S}_{3}\right)$ has recently attracted attention as an emerging functional material in nano morphologies. This metal chalcogenide compound has been shown to demonstrate a range of useful properties for applications in photodetectors, ${ }^{1-5}$ ENREF_1_ENREF_1_ENREF_1 photocatalysts, ${ }^{6-8}$ ENREF_ 5 thin film electrodes, ${ }^{9}$ gas sensors, ${ }^{10,11}$ thermoelectrics, ${ }^{12-14}$ ENREF_9 ' lithium ion batteries $^{15}$ ENREF_10and solar cells. ${ }^{16,17}$

To date, the reports on $\mathrm{Bi}_{2} \mathrm{~S}_{3}$ have mostly been focused on the fully stoichiometric n-type phase of this material. Na-

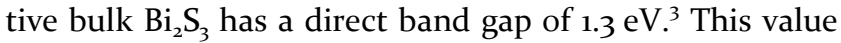
can be tuned by altering the dimensions of the material, increasing to values as high as $2.0 \mathrm{eV}$ for $\mathrm{Bi}_{2} \mathrm{~S}_{3}$ nanoparticles. ${ }^{18}$ In a nano ribbon morphology, this material has shown a photoresponsitivity exceeding $4 \mathrm{~A} / \mathrm{W}$, resulting in a quantum efficiency of above $850 \%{ }^{1} \mathrm{Bi}_{2} \mathrm{~S}_{3}$ based $\mathrm{H}_{2}$ gas sensors have been demonstrated with a response time in the order of several hours at room temperature. ${ }^{10}$ For electronics, the electron mobility has been reported to be in the range of 0.3 to $0.6 \mathrm{~cm}^{2} / \mathrm{Vs}$, with on/off ratios of $10^{2}$ -
$10^{3} .^{3}$ However, to date there is no report on any other stoichiometric or sub-stoichiometric bismuth sulfide showing p-type behavior.

The most common crystal structure of stoichiometric $\mathrm{Bi}_{2} \mathrm{~S}_{3}$ is orthorhombic, with a layered structure made of atomic scale ribbons projected in the direction of the baxis, which are held together by van der Waals forces (see Figure 1). ${ }^{19}$ This favors formation of one-dimensional nanostructures, with many previous reports of synthesized nanowires, ${ }^{3,} 5,20$ nanorods ${ }^{13,}{ }^{21}$ and nanotubes. ${ }^{19}$ ENREF 3 Only one previous report of a twodimensional $\mathrm{Bi}_{2} \mathrm{~S}_{3}$ structure has been presented by Chen et al. ${ }^{1}$ In their work, $\mathrm{Bi}_{2} \mathrm{~S}_{3}$ nanoribbons were synthesized using chemical approaches to form quasi twodimensional structures. The existence of $\mathrm{Bi}_{2} \mathrm{~S}_{3}$ in nanoribbon morphology is an indication that this material can possibly be exfoliated from its highly ordered bulk crystals into larger, planar nanosheets.

It has been widely established that two-dimensional forms of materials such as graphene, phosphorene and transition metal dichalcogenides show vastly altered optical and electronic properties from their bulk counterparts. ${ }^{22-26}$ ENREF_13 We predict that such phenomena 
can also be observed in two-dimensional bismuth sulfides,

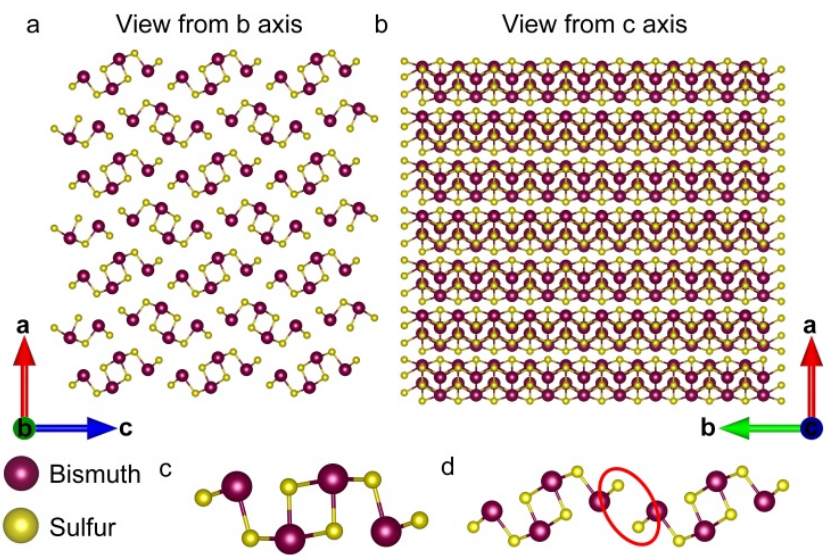

Figure 1: crystal structure of $\mathrm{Bi}_{2} \mathrm{~S}_{3},{ }^{27}$ a) view from the $b$ axis showing the cross-section of the individual ribbons, b) view from the $\mathrm{c}$ axis highlighting the layered structure, c) structure of one individual $\mathrm{Bi}_{2} \mathrm{~S}_{3}$ unit and d) structure of two neighboring $\mathrm{Bi}_{2} \mathrm{~S}_{3}$ ribbons. The red circle indicates the location where the loss of one sulfur atom could lead to the cross-linking of individual ribbons resulting in sheet formation, as discussed in the context of the XPS analysis.

efficient exfoliation is achieved. To the best of our knowledge, there is no report of top-down exfoliation processes or formation of micron-sized ultrathin $\mathrm{Bi}_{2} \mathrm{~S}_{3}$ sheets. Isolation of ultrathin sheets from orthorhombic $\mathrm{Bi}_{2} \mathrm{~S}_{3}$ could present a range of interesting, previously unexplored dimension-dependent properties.

The bulk crystal of orthorhombic $\mathrm{Bi}_{2} \mathrm{~S}_{3}$ displays favored cleavage along the (o10) plane, since the planar layers are held together by weak van der Waals forces. ${ }^{28}$ Zhang et al. also report that the (040) plane has the lowest surface energy out of the major crystal facets. ${ }^{16}$ As a result, we predict that it is likely that successful exfoliation of $\mathrm{Bi}_{2} \mathrm{~S}_{3}$ would occur parallel to this plane. However, harsh exfoliation conditions could possibly result in the breakdown of the planar arrangement of the ribbon-like units, leading to the exfoliation of one dimensional structures. Hence the exfoliation strategy has to be carefully designed in order to maintain the desired planar morphology.

A variety of liquid phase exfoliation approaches have been investigated for layered materials including graphene and transition metal dichalcogenides. ${ }^{29-34}$ ENREF_16 Many of such liquid exfoliation techniques are based on processes such as the intercalation of the layered entity using a highly reactive lithium reagent, ${ }^{35-37}$ probe sonication in a surfactant solution ${ }^{29,} 3^{0,} 3^{8}$ or a low energy solvent, ${ }^{32}$ and delaminating the layered material by reaction with a reducing agent like hydrazine. ${ }^{39,} 4^{\circ}$

Out of such techniques, the hydrazine reduction process has distinct advantages. It is a very effective technique, requiring a short processing time and, more importantly, no mechanical agitation. Consequently, successful exfoliation can occur without resulting in the breakdown of the exfoliated sheets. As a result, ultrathin sheets of exfoliated material with very large lateral dimensions can be obtained. The hydrazine reduction process has recently been demonstrated for the exfoliation of $\mathrm{MoS}_{2}$ by our group. ${ }^{4^{\circ}}$ We hypothesize that it can also be used for the exfoliation of large sheets from highly ordered bismuthinite, the naturally abundant crystals of $\mathrm{Bi}_{2} \mathrm{~S}_{3}$.

In this work, we exfoliate highly ordered $\mathrm{Bi}_{2} \mathrm{~S}_{3}$ crystals, using a hydrazine salt, to obtain large sheets of twodimensional bismuth sulfide. The chemical exfoliation process eliminates the need for sonication of the reaction mixture and results in large sheets, despite the fact that native $\mathrm{Bi}_{2} \mathrm{~S}_{3}$ is comprised of in-plane van der Waals ribbons. The sheets are fully characterized to reveal their stoichiometry and morphological properties. Subsequently, devices based on the exfoliated nanosheets are established to investigate their electrical and sensing properties.

\section{Experimental Section}

Materials and Methods. Bulk $\mathrm{Bi}_{2} \mathrm{~S}_{3}$ powder (99.9\%) was purchased from Strem. Hydrazine dihydrochloride (98.5\%) and dimethylformamide (DMF) (99.8\%) were purchased from Sigma Aldrich.

Reductive exfoliation. The method used for the exfoliation of stratified $\mathrm{Bi}_{2} \mathrm{~S}_{3}$ was similar to that recently developed by our group for exfoliating $\mathrm{MoS}_{2}{ }^{40} \mathrm{o} .5 \mathrm{~g}$ of hydrazine dihydrochloride and $0.1 \mathrm{~g}$ of bismuth sulfide were mixed into $10 \mathrm{~mL}$ of DMF and heated to reflux at $153^{\circ} \mathrm{C}$ for 1 hour, with constant stirring. The mixture was then stored at $-30^{\circ} \mathrm{C}$ for approximately 90 minutes, to solidify remaining organic side products. The solution was centrifuged at 200 RCF for 15 minutes to remove unreacted bulk $\mathrm{Bi}_{2} \mathrm{~S}_{3}$. The produced suspension was then centrifuged at 16,0oo RCF for 6o minutes to sediment out the ultrathin bismuth sulfide. The ultrathin sheets were re-suspended and washed several times using fresh DMF. We avoided washing with water since the addition of water leads to the crystallization of large amounts of bismuth oxychloride $(\mathrm{BiOCl})$ which forms from excess dissolved bismuth and chloride ions (See Figure $\mathrm{S} 1$ ).

Device Fabrication. The suspended nanosheets were drop casted onto highly p-doped $290 \mathrm{~nm} \mathrm{SiO}_{2} / \mathrm{Si}$ substrates pre-patterned with a registration scheme of $\mathrm{Au}$ markers. Optical and scanning electron microscopy (SEM) images were subsequently used to locate suitable sheets. Electrodes were patterned using electron beam lithography (EBL) at $25 \mathrm{keV}$ (FEI XL40 SEM) using the substrate markers for the alignment, followed by deposition of $10 \mathrm{~nm} \mathrm{Ti}$ and $50 \mathrm{~nm} \mathrm{Au} \mathrm{using} \mathrm{electron} \mathrm{beam} \mathrm{evap-}$ oration.

Characterization. The suspension of ultrathin sheets in DMF was dried on silicon substrates, glass slides and transmission electron microscopy (TEM) grids for further analysis. TEM was performed on a JEOL 1010 instrument $(100 \mathrm{keV})$, to determine the lateral dimensions of the sheets. High resolution TEM (HRTEM) images were collected using a JEOL 2100 F instrument (200 keV). SEM was conducted using an FEI Verios instrument operating with 
a landing energy of $1 \mathrm{kV}$ (stage bias $4 \mathrm{kV}$ ). Optical images were collected using a Leica DM250oM microscope with a CCD camera. The thickness of the sheets was determined using a Bruker Dimension Icon atomic force microscope (AFM) in tapping mode.

A map of photoluminescence spectra was obtained using a Horiba Scientific LabRAM HR evolution system with $5 \mathrm{~s}$ exposure using excitation wavelength of $532 \mathrm{~nm}$. A Reinshaw inVia spectrometer with laser excitation of $785 \mathrm{~nm}$ was employed to collect the Raman spectrum of the material. The powder x-ray diffraction (XRD) pattern of the sheets was collected using a Bruker $\mathrm{D}_{4}$ Endeavour with $\mathrm{Cu} \mathrm{K} \alpha$ radiation of $1.5406 \AA$.

The elemental composition was studied by $\mathrm{x}$-ray photoelectron spectroscopy (XPS) using a Thermo Scientific Kalpha instrument with a monochromated $\mathrm{Al} \mathrm{K \alpha}$ x-ray source $(1486.7 \mathrm{eV})$. The bulk material was also studied using an Oxford Instruments X-Max 8o energy dispersive $\mathrm{x}$-ray spectroscopy (EDS) detector connected to the SEM.

Electrical measurements were performed using a Keithley 2400 sourcemeter. Standard 2-probe DC techniques were used, at ambient temperature and pressure, with a back gate voltage applied to the highly p-doped Si substrate. Elevated temperature measurements were achieved using a Linkam stage, with a temperature controlled stub.

Photoelectron spectroscopy in air (PESA) was used for determining the valence band position of samples dried on glass substrates, using a Riken Kekei AC-2.

Gas sensing was achieved using a custom sensing chamber attached to a digital mass flow controller, which was set at a flow rate of $200 \mathrm{sccm}$. The $\mathrm{NO}_{2}$ concentration was 10 parts per million (ppm) in background air. $\mathrm{H}_{2} \mathrm{~S}$ and $\mathrm{H}_{2}$ were set to $56 \mathrm{ppm}$ in $\mathrm{N}_{2}$ and $1 \%$ in air, respectively. Water vapor was introduced at 50\% relative humidity.

\section{Results and Discussion}

\section{Structure and Morphology}

Liquid phase exfoliation of bulk $\mathrm{Bi}_{2} \mathrm{~S}_{3}$ was carried out, as presented in the Experimental section, and the remaining bulk powder was removed. In brief, we used hydrazine dihydrochloride facilitated exfoliation, which does not require any mechanical agitation and leads to nanosheets of large lateral dimensions. After centrifugation, large amounts of grey sediment were observed, which indicates that there is a high yield of ultrathin material produced. These were washed and re-suspended in DMF for further analysis.

The suspended nanosheets were studied by several techniques, to determine their size and morphology. 30 different sheets on a grid were measured by TEM, as presented in the size histogram (Figure 2a). A range of micron-scale dimensions are observed, with the majority of sheets being 2-4 $\mu \mathrm{m}$ in length, and some reaching dimensions as large as $20 \mu \mathrm{m}$. The highly wrinkled nature of the sheets when dried upon the TEM grid suggests that the true dimensions, when laid flat, would be even larger. It seems that the lateral dimension is limited by the dimensions of the starting bulk powder. a
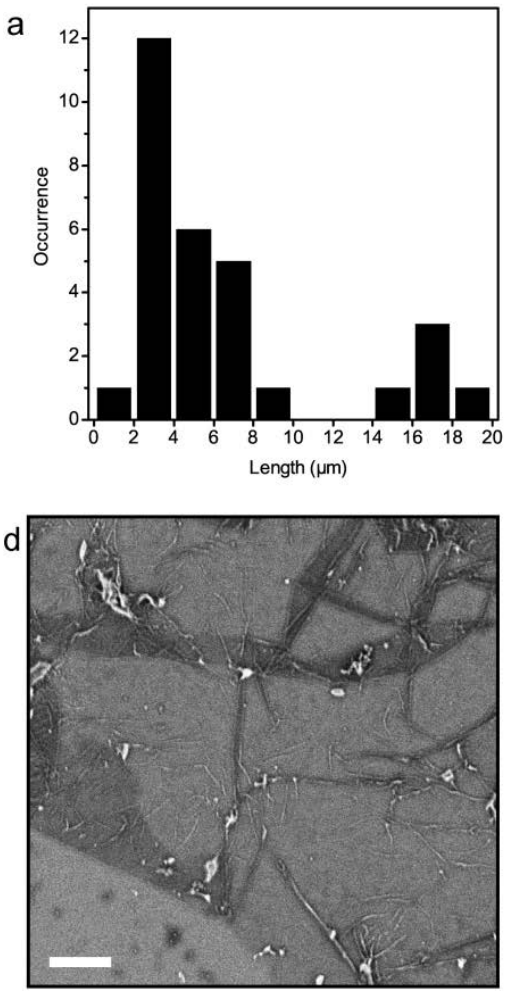
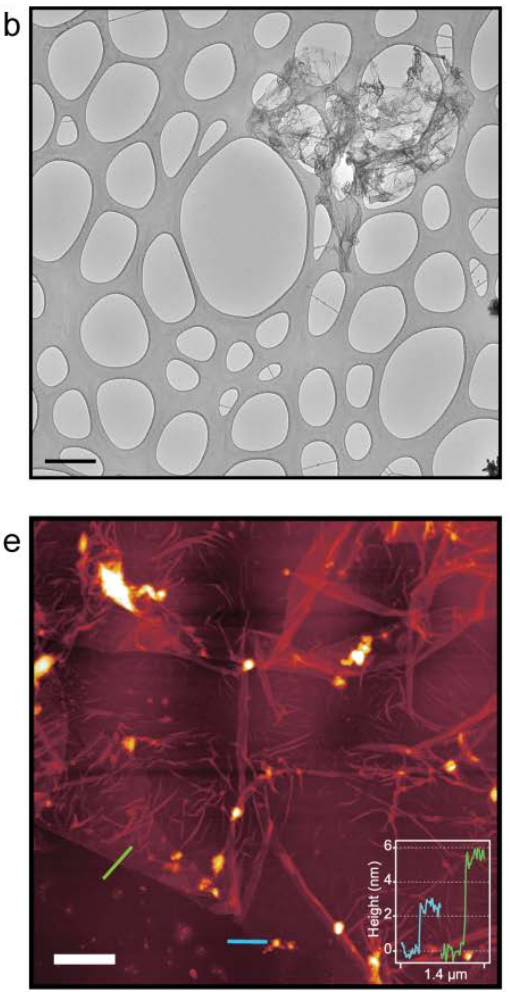

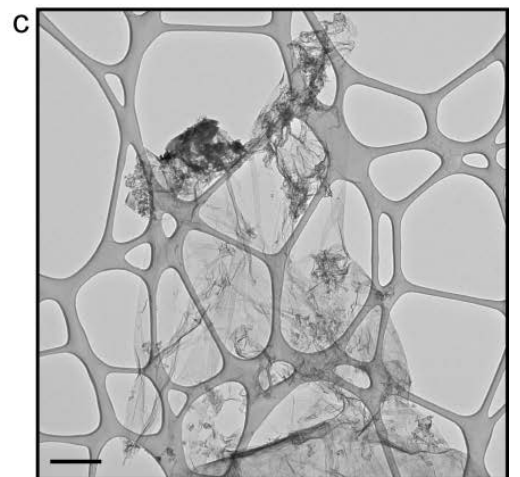

$f$

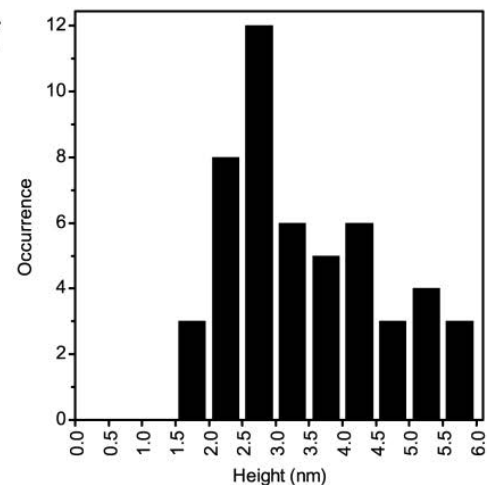

Figure 2. a) Size histogram of 30 sheets observed by TEM. b) TEM image of a small sheet, scale bar $0.5 \mu \mathrm{m}$. c) TEM image of a large sheet, scale bar $0.5 \mu \mathrm{m}$. d) SEM image of the edge of a sheet, scale bar $1 \mu \mathrm{m}$. e) AFM image of the same area shown in d), 
scale bar $1 \mu \mathrm{m}$. Inset height profiles of the blue and green lines. $\mathrm{f}$ ) Thickness histogram prepared by measuring 50 flat and folded edges.

Example TEM images of a small and large sheet are presented in Figure $2 \mathrm{~b}$ and $\mathrm{c}$, additional TEM images with different degrees of wrinkling are presented in Figure S2. Transparent regions, where the sheets lay flat, indicate that the product is expected to be ultrathin. Additionally, the flexibility of the sheets is always associated with the low number of fundamental layers forming an ultrathin sheet. $^{41,42}$

SEM imaging was used for studying the structure of the sheets further, using settings to enhance the surface detail, enabling the observation of wrinkles and thin edges. Differences in the contrast of the SEM images show where the edge of the sheet lays flat or is folded over (See Figure 2d). It is also observed that some thick species have dried onto the sheet. These could be small pieces of unexfoliated material or other insoluble impurities and side products. Further optimization of the washing process is necessary to obtain perfectly clean, uniform samples.

The thickness of the sheets was confirmed using AFM measurements (Figure ze and $\mathrm{f}$ ) which show that a step at the flat edge of a sheet is $2.5 \mathrm{~nm}$. The thickness of a full stoichiometric monolayer would be limited by the lattice parameter of $1.1 \mathrm{~nm} .{ }^{1}$ We were not able to find any step of this thickness in our samples as can be seen in Figure $2 f$. The minimum observed thickness was found to be just above $1.5 \mathrm{~nm}$ in all our observations (Figure $2 \mathrm{f}$ ). We will associate this increase of the thickness with the development of a corrugated plane structure that will be discussed in the context of HRTEM imaging later in this manuscript. Many folded edges are also observed, as shown by the green trace in Figure 2e, featuring a bilayer thickness in the order of $5 \mathrm{~nm}$. Additional AFM images are also presented in Figure $\mathrm{S}_{3}$.

As an example, a single sheet was analyzed by a variety of techniques (SEM, AFM, optical images and photoluminescence), to determine the combined information that can be obtained for a single sheet (Figure $\mathrm{S}_{4}$ ). The observed folds and wrinkles in the material not only confirm the thinness of the sheets, but also show their strength and flexibility, since there are no signs of fracturing along the folds.

To further analyze the thickness of the exfoliated sheets, HRTEM was used, as presented in Figure 3. In the low magnification image, a single layer with several folds is observed (Figure za inset). Analyzing folded edges of the nanosheets allows gaining insight into the local surface morphology of the nanosheets which can otherwise not be observed for flat laying regions. Higher magnification shows that the folded edges follow a zig-zag pattern (Figure $3 a$ and $b$ ). This pattern is consistent with the crystal structure of orthorhombic $\mathrm{Bi}_{2} \mathrm{~S}_{3}$, with an offset herringbone arrangement of repeating units. ${ }^{43}$ The height of the observed zig-zag, however, is close to $\sim 1.3 \mathrm{~nm}$ (Figure $3 \mathrm{~b}$ ), indicating that the plane has been warped into deeper corrugations. Adding the depth of the observed corrugation to the lattice parameter of a single layer $(1.1 \mathrm{~nm})$ al- lows establishing an approximation for the expected
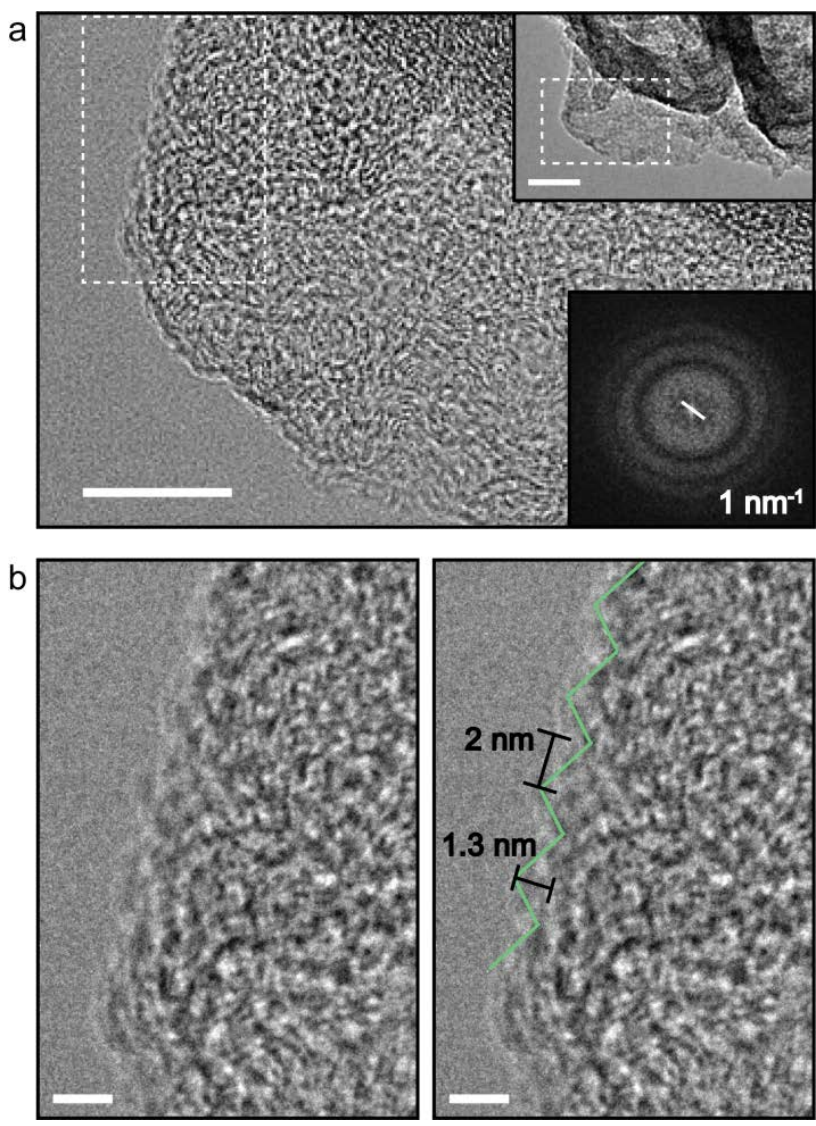

Figure 3. a) HRTEM of a nanosheet, scale bar $10 \mathrm{~nm}$. Inset top, lower magnification, scale bar $20 \mathrm{~nm}$. Inset bottom, Fourier transform pattern showing spots at $1 \mathrm{~nm}^{-1}$. b) Higher magnification of a) showing zig-zag edge with corrugations of $1.3 \mathrm{~nm}$ depth and a lateral peak-to-trough distance of $2 \mathrm{~nm}$, scale bar $2 \mathrm{~nm}$.

layer thickness of the exfoliated sheets which is found to be $\sim 2.4 \mathrm{~nm}$ for a corrugated monolayer. This number is consistent with the step height observed using AFM in Figure $2 \mathrm{f}$, indicating that monolayer bismuth sulfide is synthesized. As can be seen in Figure za the crystal lattice has been highly distorted, although some areas of localized crystallinity can still be identified.

We, hence, speculate that the reductive exfoliation process alters the in-plane crystal structure of the bismuth sulfide, by possibly introducing sulfur vacancies and allowing the ribbon-like units to interact in an alternate arrangement. The assumption regarding the presence of sulfur vacancies will be further explored using x-ray photoelectron spectroscopy (XPS) later in this manuscript. These vacancies cause a repeating unit with corrugations of $1.3 \mathrm{~nm}$ along the c-axis, resulting in a monolayer with a uniform height of $2.4 \mathrm{~nm}$ and folded edges with double this height. The spacing between the corrugations is observed to be $\sim 2 \mathbf{n m}$, and is identified as spots in the Fourier transform (Figure za inset) at a diameter of $\sim 1 \mathrm{~nm}^{-1}$. The 
observation of a repeating unit, with directionality, implies that the nanosheets would possess anisotropic properties and these will be studied in future investigations.
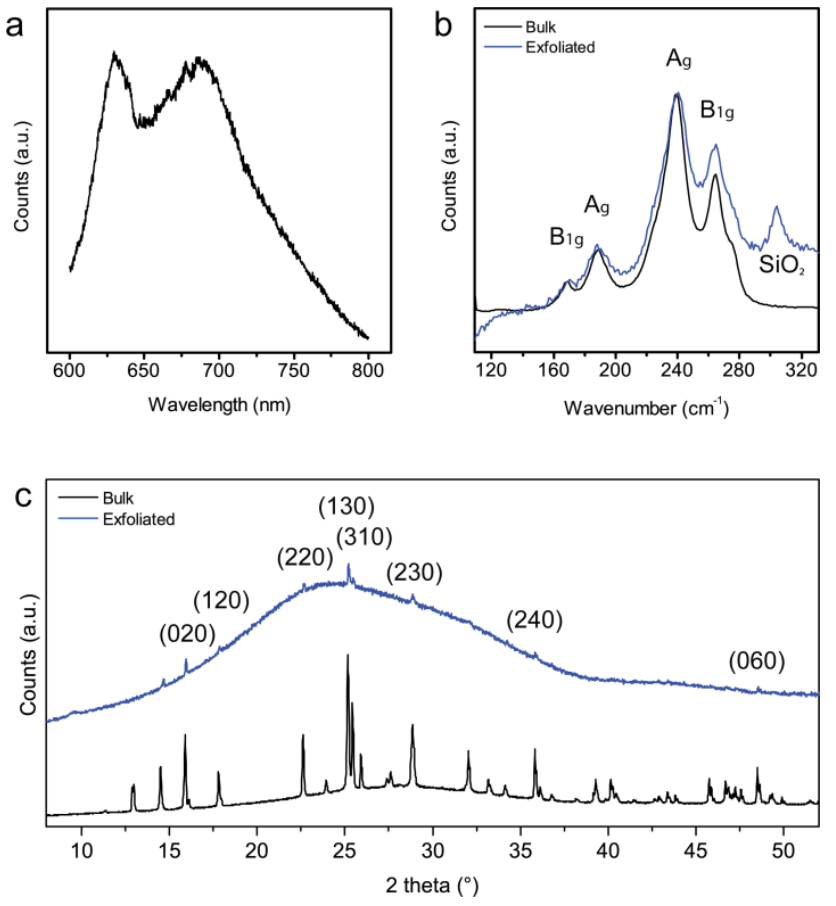

Figure 4. a) Photoluminescence spectrum of a sheet. b) Raman spectra of exfoliated and bulk material. c) XRD patterns of exfoliated and bulk material with main peaks indexed. Data has been normalized for ease of comparison.

\section{Composition}

The exfoliated sheets were characterized and compared to the bulk starting material to determine the composition of the products. Photoluminescence was observed from the sheets when a $532 \mathrm{~nm}$ laser excitation was used. The emission shows a peak centered at $629 \mathrm{~nm}$ and a second, broader peak at $689 \mathrm{~nm}$ (Figure 4a). The observed photoluminescence is in the same energy range as previously reported luminescence from $\mathrm{Bi}_{2} \mathrm{~S}_{3}$ nanobelts. ${ }^{44}$ It has been suggested that crystal defects may create deep trap states that provide alternate recombination pathways resulting in the observed photoluminescence. ${ }^{45-47}$ The intensity of the photoluminescence emission is directly proportional to the thickness of the material (see Figure $S_{4} b$ ). We do not see enhanced emission for ultrathin regions, as has been reported for other two-dimensional materials. ${ }^{48}$ This is probably due to the fact that the bulk material already possesses a direct bandgap, and thus no bandgap crossover can be observed when the thickness of the crystal is reduced.

The Raman spectra (Figure $4 \mathrm{~b}$ ) show similar patterns for bulk $\mathrm{Bi}_{2} \mathrm{~S}_{3}$ and thin layers. The peak locations closely match previously reported values, ${ }^{3}$ the transverse $A_{g}$, inplane, peaks occur at 188 and $240 \mathrm{~cm}^{-1}$, and the longitudinal $B_{1 g}$ vibrations are at 168 and $265 \mathrm{~cm}^{-1}$. ${ }^{49}$ However, the relative peak intensity of the main $B_{1 g}$ peaks is increased in the exfoliated sample, which has previously been re- ported for $\mathrm{Bi}_{2} \mathrm{~S}_{3}$ quantum dots. ${ }^{50}$ This indicates that the longitudinal vibrations are altered compared to bulk crystals of $\mathrm{Bi}_{2} \mathrm{~S}_{3}$.

a

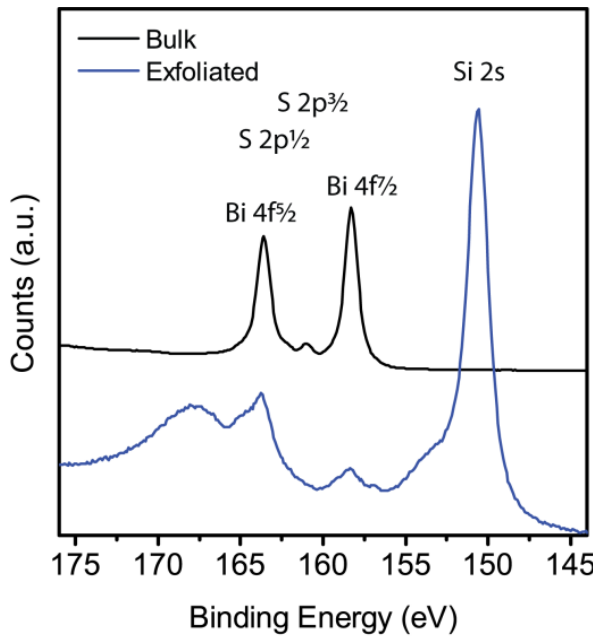

b

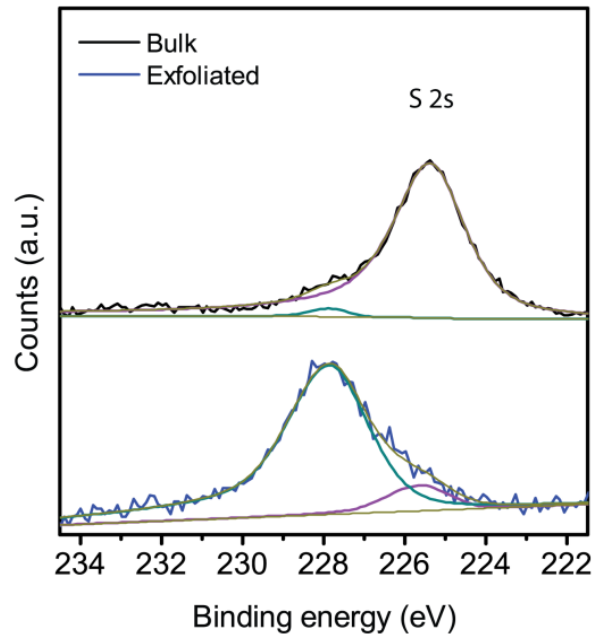

C

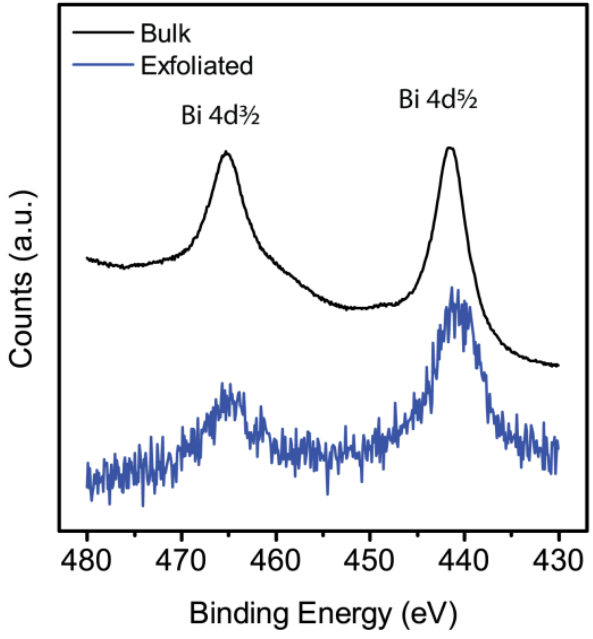

Figure 5. XPS binding energy windows for a) Bi 4f, b) S $2 \mathrm{~S}$ and c) Bi 4d, for exfoliated (blue) and bulk material (black). Data has been normalized for ease of comparison.

An exfoliated sample dried on glass shows an XRD pattern that corresponds well to the bulk powder (Figure 4c). The broad feature between $15-35^{\circ}$ is a background signal 
associated with the glass substrate. All peaks are indexed to crystal planes of orthorhombic $\mathrm{Bi}_{2} \mathrm{~S}_{3}$ (JCPDS 17-0320). The bulk material features major contributions from (130), (310) and (020) peaks. The exfoliated material is expected to show preferential orientation in the (o10) and parallel planes, which would increase the peak intensities for these directions. The absence of these intense peaks can be attributed to the highly wrinkled nature of the sheets when dried on a substrate, resulting in random orientation. Additionally, the overall peak intensities have significantly decreased for all peaks, which further confirms the distortion of the crystal lattice during the chemical exfoliation, in agreement with the HRTEM analysis.

The XPS data (Figure $5 \mathrm{a}$ ) of the bulk $\mathrm{Bi}_{2} \mathrm{~S}_{3}$ reference, sample measured for the binding energy region between 145 and $175 \mathrm{eV}$, show a Bi $4 \mathrm{f}$ doublet with the $\mathrm{S} 2 \mathrm{p}$ doublet inside the $\mathrm{Bi} 4 \mathrm{f}$ doublet gap. These peaks were fitted for the bulk material (Figure $\mathrm{S}_{5} \mathrm{a}$ ) and sensitivity factors were calibrated to yield the expected $\mathrm{Bi}$ to $\mathrm{S}$ atomic ratio of 40:6o for $\mathrm{Bi}_{2} \mathrm{~S}_{3}$. The atomic ratio of the precursor $\mathrm{Bi}_{2} \mathrm{~S}_{3}$ material was confirmed using EDS during SEM imaging, giving a ratio of $\sim 38: 62$, as shown in Figure $\mathrm{S}_{5} \mathrm{~b}$.

For the exfoliated sample (Figure 5a, blue line), broadening of the $\mathrm{Bi} 4 \mathrm{f}$ peaks to higher binding energies indicates that some Bi within the sample exists in a sulfur-deficient state. ${ }^{51}$ The stoichiometric analysis of the sample is found to be complicated due to the overlapping of the $\mathrm{Bi} 4 \mathrm{f}$ and S 2p peaks which are also found to be comparatively weak. Furthermore, interference from the substrate's Si 2s signal is observed. As a result, it is not accurate to use this energy window for peak fitting.

As an alternative, the less prominent $\mathrm{S} 2 \mathrm{~s}$ and $\mathrm{Bi} 4 \mathrm{~d}$ energy windows (Figure $5 \mathrm{~b}$ and $\mathrm{c}$ ) are used, since these peaks are found to be more defined while not being affected by any overlapping signals from other elements. The $\mathrm{S} 2 \mathrm{~S}$ peak corresponding to elemental sulfur at $227.9 \mathrm{eV}$ (green line) and the $\mathrm{Bi}_{2} \mathrm{~S}_{3} \mathrm{~S} 2 \mathrm{~S}$ peak at $225.6 \mathrm{eV}$ (purple line) are fitted, and the peak corresponding to $\mathrm{Bi}_{2} \mathrm{~S}_{3}$ is used for the atomic ratio calculation. ${ }^{6,28,52,53}$ Oxidation of the material is ruled out by closer observation of the $\mathrm{O}$ is region and extended Raman spectrum (Figure S6). The Raman spectrum does not show any evidence of peaks at 450 or 530 $\mathrm{cm}^{-1}$ which would indicate the presence of $\mathrm{Bi}_{2} \mathrm{O}_{3}$. Moreover, the symmetrical $\mathrm{O}$ is peak is assigned to the $\mathrm{SiO}_{2}$ substrate with no evidence of a shoulder or peak at higher energy levels, which would be observed for $\mathrm{Bi}_{2} \mathrm{O}_{2} \mathrm{~S}$ and $\mathrm{Bi}_{2} \mathrm{O}_{3}{ }^{56-58}$ The $\mathrm{Bi} 4 \mathrm{~d}$ doublet is also integrated and the atomic ratio between $\mathrm{Bi}$ and $\mathrm{S}$ in the exfoliated material is found to be 43:57, corresponding to a chemical formula of $\mathrm{Bi}_{2} \mathrm{~S}_{2.6}$. This indicates that the product is not fully stoichiometric $\mathrm{Bi}_{2} \mathrm{~S}_{3}$, but rather that there is a large amount of sulfur vacancies introduced to the sheets during the exfoliation process. The lost sulfur atoms are likely reduced to elemental sulfur during the hydrazine based exfoliation procedure, giving rise to the associated distinct XPS signal (Figure 5b) and potentially the observed residues mentioned in the SEM discussion.

The determined elemental ratio indicates that most fundamental building blocks of the ribbon structure (Figure 1c) have lost one sulfur atom. The loss of one sulfur atom per bismuth sulfide unit provides a suitable hypothesis for the origin of the observed corrugated $2 \mathrm{D}$ sheet formation. If the sulfur loss preferentially involved the terminal sulfur atoms (highlighted in Figure $1 \mathrm{~d}$ ), cross linking of the individual ribbons and $2 \mathrm{D}$ sheet formation can be rationalized due to the formation of sulfur bridges. The van der Waals gap in c-axis direction is only $3.16 \AA$ wide while the distance between the nanoribbons in the a axis is $3.45 \AA$. The sulfur to bismuth bond lengths in bulk $\mathrm{Bi}_{2} \mathrm{~S}_{3}$ are found to be between $2.5 \AA$ and $3.0 \AA$ and predominantly between $2.5 \AA$ and $3.2 \AA$ in simulated quantum confined $\mathrm{Bi}_{2} \mathrm{~S}_{3}$ systems, indicating that $2 \mathrm{D}$ cross linking rather than the formation of a three dimensional system is preferred. ${ }^{59}$ As previously discussed, these vacancies, in combination with the formation of sheets, are the possible causes of the in-plane corrugation.

The presence of $\mathrm{Bi}$ ions in the reaction mixture (as indicated in the Experimental section by the formation of $\mathrm{BiOCl}$ ) along with the altered atomic ratio, suggests that the harsh reaction conditions cause some breakdown of the material during the exfoliation process. This resulting substoichiometry is consistent with previous characterization of hydrazine assisted exfoliation of $\mathrm{MoS}_{2} \cdot{ }^{40}$

\section{Electrical Properties}

Back-gated devices were established to facilitate the electrical characterization of the exfoliated sheets. Optical and SEM imaging (Figure $\mathrm{S}_{7}$ ) were employed to locate the dispersed nanosheets on a substrate after drop casting the suspension. Large sheets that lay reasonably flat on the pre-patterned $\mathrm{SiO}_{2} / \mathrm{Si}$ substrate are selected for electrode deposition. The thinnest and flattest part of the sheets were identified and measured with respect to the markers and EBL was used to pattern the electrodes, an example of which is shown in Figure 6a. A total of four operational structures was fabricated which displayed consistent properties. The location was then studied by AFM (Figure 6b) to determine the thickness of the sheet, wrinkles and also to confirm the height of the Au/Ti electrodes. The sheet was found to be $\sim 3 \mathrm{~nm}$ thick, with wrinkles reaching $6 \mathrm{~nm}$ of height (Figure 6c). This indicates that the electrical measurements were collected for single layer sheets.

The devices were measured with various applied backgate voltages and the current-voltage (I-V) curves were collected across the drain (an example is presented in Figure $6 \mathrm{~d}$ ). Non-ohmic contacts are observed, likely due to the chemical processing of the nanosheets, resulting in mobilities limited to the order of $10^{-3} \mathrm{~cm}^{2} / \mathrm{Vs}$. The enhanced current at negative gate voltage suggests that the sample has p-type nature. $\mathrm{Bi}_{2} \mathrm{~S}_{3}$ is usually reported as an n-type material, ${ }^{14}$, 6o and this $\mathrm{p}$-doping is likely due to altered properties resulting from chemical preparation of the sheets and the resulting substochiomety. The sample displays weak transconductance and does not switch off at gate voltages up to $80 \mathrm{~V}$, where leakage typically occurs, indicating metallic doping of the material. Reports of metal-like conduction in substoichiometric $\mathrm{Bi}_{2} \mathrm{~S}_{3}$ have attributed this phenomenon to sulfur vacancies in the 
material. ${ }^{13,61,62}$ Annealing substoichiometric $\mathrm{Bi}_{2} \mathrm{~S}_{3}$ in a sulfur atmosphere was shown to decrease the electrical conductivity by reducing the carrier concentration, as a result of the reduced number of sulfur vacancies. ${ }^{62}$ In order to

a

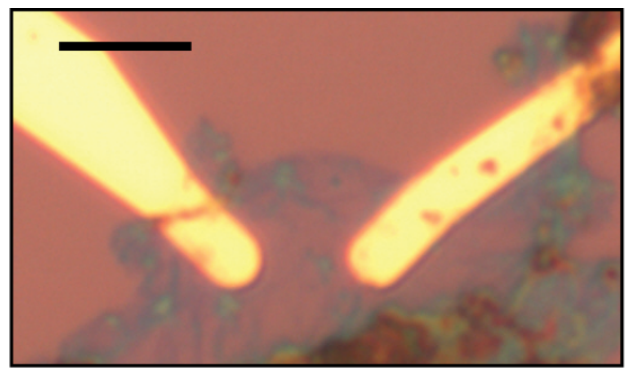

b
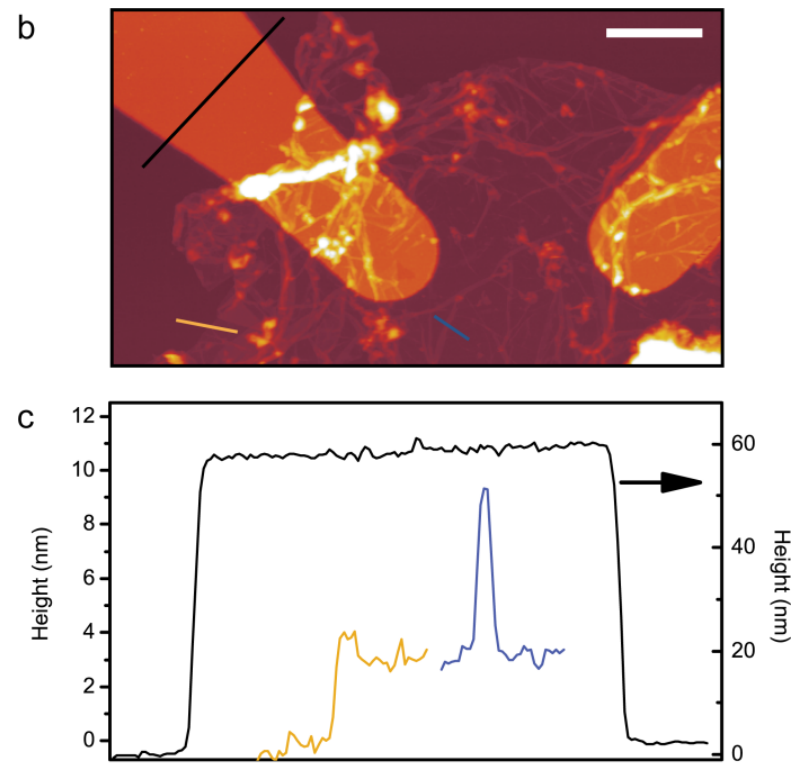

d
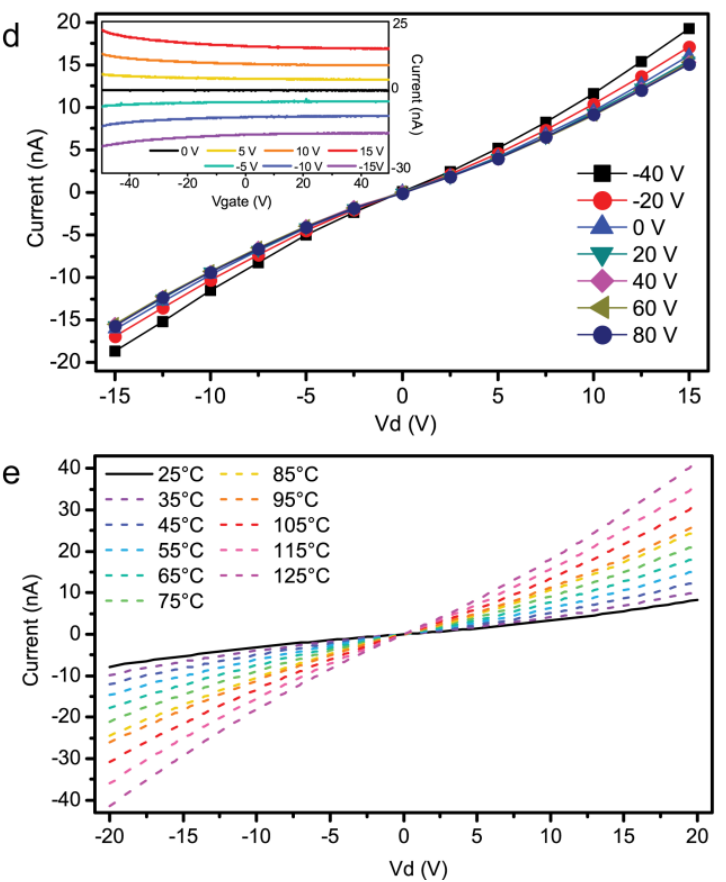

Figure 6. Electrodes deposited on an exfoliated nanosheet. a) Optical image, scale bar $5 \mu \mathrm{m}$. b) AFM image of the same device, scale bar $2 \mu \mathrm{m}$. c) Height profiles of lines in b), showing thicknesses of the sheet, wrinkles and electrodes. d) I-V curves of device using various applied gate voltages. Inset, IVgate curves of device with various drain voltages. e) I-V curves of device at various temperatures.

improve the materials performance in logic devices, more work should be carried out, to improve the contacts and to also tune the material's sulfur content in order to obtain a higher mobility in this p-type material.

To further investigate the electrical properties of the nanosheets, the valence band position and Fermi level of the material were determined using PESA and valence XPS investigation (Figure S8). PESA found the material's valence band position to be $5.2 \mathrm{eV}$. This is consistent with the reported value of $5.13 \mathrm{eV}$, calculated for the (o10) surface of $\mathrm{Bi}_{2} \mathrm{~S}_{3} .{ }^{63}$ The extremely small gap between the Fermi level and the valence band $(\sim 0.1 \mathrm{eV})$ indicates the presence of excess holes in the valence band and is indicative of high p-doping, which is in agreement with the electrical measurements.

The device's response to elevated temperature was also studied. I-V curves collected from $25^{-12} 5^{\circ} \mathrm{C}$ (Figure 6e) show that the current increases near-linearly with increasing temperature. This translates into a negative temperature co-efficient where the resistivity changes from $\sim 290$ to $\sim 55 \Omega \mathrm{cm}^{-1}$ when the temperature is increased from $25^{\circ} \mathrm{C}$ up to $125^{\circ} \mathrm{C}$. This order of change in resistivity with temperature is comparable to that reported previously for n-type $\mathrm{Bi}_{2} \mathrm{~S}_{3}{ }^{13}$ The change in resistivity is relatively linear which means that the device can be efficiently used for room temperature sensing applications.

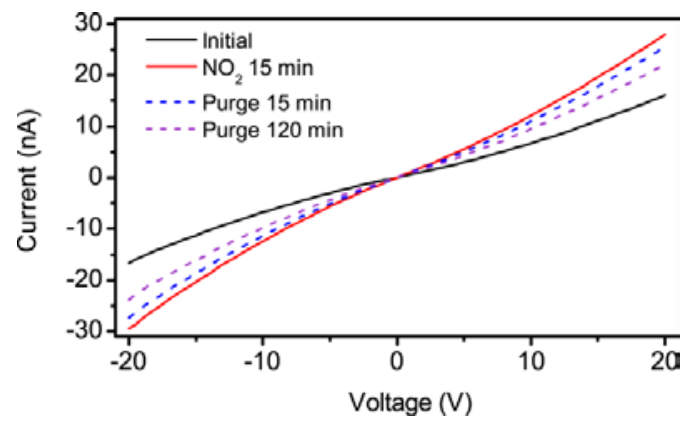

Figure 7. I-V curve of device before (black) and after (red) exposure to $\mathrm{NO}_{2}$ gas for 15 minutes at room temperature and subsequent purging (dashed).

\section{Gas Sensing}

The device was also investigated for the use as a room temperature gas sensor. The I-V curve was collected, at room temperature, before and after exposure to $10 \mathrm{ppm}$ $\mathrm{NO}_{2}$ for 15 minutes (Figure 7 ). The I-V curve indicates an increased conductivity of the nanosheet, leading to an increased current by a response factor of 1.8 after exposure to $\mathrm{NO}_{2}$ gas. The increase in conductivity with adsorbed $\mathrm{NO}_{2}$ is observed since the material is a p-type semiconductor and $\mathrm{NO}_{2}$ acts as an electron acceptor. ${ }^{64}$ 
The sensor shows a fast response, but a slow recovery, as highlighted in Figure 9 $_{9}$.

After purging the sample for two hours with synthetic air, the device still showed an elevated conductivity. This slow recovery time indicates that the gas molecules strongly interact with the device and the adsorption is not quickly reversed. However, when measured after resting overnight, the device completely returned to its original state, which shows that no permanent bonding between the gas and the device occurred and that the nature of the interaction is probably physisorption. Similar slow recovery times are widely reported for other forms of physisorption based $\mathrm{NO}_{2}$ sensors including two-dimensional $\mathrm{MoS}_{2}$ and carbon based devices. ${ }^{65,66}$

$\mathrm{Bi}_{2} \mathrm{~S}_{3}$ nanowires and $\mathrm{WS}_{2}$ nanosheets have shown promise as humidity sensors. ${ }^{67,68}$ As such, the sensor's response to water vapor was also investigated, to determine if the humidity of the environment will interfere with the sensor's response to $\mathrm{NO}_{2}$ gas. The current signal was found to decrease on the transition from a dry environment to $50 \%$ relative humidity, as shown in Figure Sro. This indicates that the sensor may show a weaker signal when measured in a humid environment, but the humidity will not interfere to give a false-positive response to $\mathrm{NO}_{2}$ gas.

A previous report on gas sensing using a $\mathrm{Bi}_{2} \mathrm{~S}_{3}$ nanowire shows moderate response and recovery time for interaction with $\mathrm{H}_{2}$ gas. ${ }^{10}$ As such, our sensor was also exposed to $\mathrm{H}_{2}$ and $\mathrm{H}_{2} \mathrm{~S}$ gases, however, no response was detected (Figure S11). This discrepancy is attributed to the way the gases interact with the two different materials. Yao et al. ${ }^{10}$ use n-type one-dimensional nanowires and do not report sulfur vacancies in their material. Hence, it is predicted that our material does not interact with $\mathrm{H}_{2}$ due to the different structure of the used bismuth sulfide based materials. Furthermore, these measurements indicate that substoichiometric p-type nanosheets have a good selectivity for $\mathrm{NO}_{2}$ gas sensing.

\section{Conclusion}

A new two-dimensional form of bismuth sulfide was developed, using a hydrazine salt based liquid phase exfoliation process. Since no mechanical force was required during this process, micron-scale ultrathin corrugated nanosheets could be obtained, despite the fact that the ribbon-like entities are held together by weak van der Waals forces along the planar structures in the bulk sample. The synthesized corrugated sheets were as large as $20 \mu \mathrm{m}$ across and as thin as $2.5 \mathrm{~nm}$. Sulfur vacancies introduced during the exfoliation process altered the stoichiometry of the material, resulting in a chemical formula of $\mathrm{Bi}_{2} \mathrm{~S}_{2.6}$. The loss of sulfur atoms likely facilitated the cross linking of the individual bismuth sulfide ribbons, leading to the formation and exfoliation of corrugated $2 \mathrm{D}$ nanosheets. Further work on the characterization of the highly anisotropic material is expected to show interesting altered properties along the corrugations. A metallic electrical response, with weak transconductance, was displayed and hence, future studies will investigate means to control the sulfur content to tune the material's electron- ic properties. A near-linear response to temperatures from $25-125^{\circ} \mathrm{C}$ highlights the potential use of this material in temperature measurement systems. The material also showed promise as a selective $\mathrm{NO}_{2}$ gas sensor, with a fast response time and a response factor of 1.8. This is a first report on hydrazine salt assisted exfoliation of a highly ordered compound into corrugated planes, despite the fact that the precursor crystal was not naturally made of uniform covalently bound fundamental planes. The method can be adopted for the formation of future planar morphologies from crystals that are only quasi-stratified and, hence, lead to many novel possibilities in exfoliation processes for new two-dimensional materials.

\section{ASSOCIATED CONTENT}

Supporting Information. Additional TEM, AFM, multimodal imaging of a single nanosheet, XPS and EDS analysis of the bulk $\mathrm{Bi}_{2} \mathrm{~S}_{3}$ precursor, additional XPS and Raman data, low magnification optical and SEM images, PESA and XPS valence analysis and additional gas sensing data are located in the Supporting Information. This material is available free of charge via the Internet at http://pubs.acs.org.

\section{AUTHOR INFORMATION}

\section{Corresponding Authors:}

*Email: Kourosh.Kalantar@rmit.edu.au

*Email: Torben.Daeneke@rmit.edu.au

\section{Author Contributions}

The manuscript was written through contributions of all authors and all authors have given approval to the final version of the manuscript.

\section{ACKNOWLEDGMENT}

This work was performed at the RMIT University MicroNano Research Facility (MNRF), the Melbourne Centre for Nanofabrication (MCN), in the Victorian node of the Australian National Fabrication Facility (ANFF) and the RMIT Microscopy and Microanalysis Facility (RMMF) at RMIT University linked lab of the Australian Microscopy and Microanalysis Research Facility (AMMRF). The authors would like to acknowledge the technical assistance provided by staff in each of these facilities. B. W. acknowledges an ARC DECRA fellowship under grant number DE160101334 and K. K-z. acknowledges an ARC Discovery grant with grant number DP140100170.

\section{REFERENCES}

1. Chen, G.; Yu, Y.; Zheng, K.; Ding, T.; Wang, W.; Jiang, Y.; Yang, Q. Fabrication of Ultrathin $\mathrm{Bi}_{2} \mathrm{~S}_{3}$ Nanosheets for HighPerformance, Flexible, Visible-NIR Photodetectors. Small 2015, 11, 2848-2855.

2. Li, R.; Yang, J.; Huo, N.; Fan, C.; Lu, F.; Yan, T.; Wei, Z.; Li, J. Effect of Electrical Contact on the Performance of $\mathrm{Bi}_{2} \mathrm{~S}_{3}$ Single Nanowire Photodetectors. ChemPhysChem 2014, 15, 2510-2516.

3. Lu, F.; Li, R.; Li, Y.; Huo, N.; Yang, J.; Li, Y.; Li, B.; Yang, S.; Wei, Z.; Li, J. Improving the Field-Effect Performance of $\mathrm{Bi}_{2} \mathrm{~S}_{3}$ Single Nanowires by an Asymmetric Device Fabrication. ChemPhysChem 2015, 16, 99-103. 
4. Li, R.; Yue, Q.; Wei, Z. Abnormal low-temperature behavior of a continuous photocurrent in $\mathrm{Bi}_{2} \mathrm{~S}_{3}$ nanowires. J. Mater. Chem. C 2013, 1, 5866-5871.

5. Bao, H.; Cui, X.; Li, C. M.; Gan, Y.; Zhang, J.; Guo, J. Photoswitchable Semiconductor Bismuth Sulfide $\left(\mathrm{Bi}_{2} \mathrm{~S}_{3}\right)$ Nanowires and Their Self-Supported Nanowire Arrays. J. Phys. Chem. C 2007, 111, 12279-12283.

6. Gao, X.; Huang, G.; Gao, H.; Pan, C.; Wang, H.; Yan, J.; Liu, Y.; Qiu, H.; Ma, N.; Gao, J. Facile fabrication of $\mathrm{Bi}_{2} \mathrm{~S}_{3} / \mathrm{SnS}_{2}$ heterojunction photocatalysts with efficient photocatalytic activity under visible light. J. Alloys Compd. 2016, 674, 98-108.

7. Zhang, Z.; Wang, W.; Wang, L.; Sun, S. Enhancement of Visible-Light Photocatalysis by Coupling with Narrow-Band-Gap Semiconductor: A Case Study on $\mathrm{Bi}_{2} \mathrm{~S}_{3} / \mathrm{Bi}_{2} \mathrm{WO}_{6}$. ACS Appl. Mater. Interfaces 2012, 4, 593-597.

8. Wu, T.; Zhou, X.; Zhang, H.; Zhong, $\mathrm{X} . \mathrm{Bi}_{2} \mathrm{~S}_{3}$ nanostructures: A new photocatalyst. Nano Res. 2010, 3, 379-386.

9. Tamašauskaitė-Tamašiūnaitè, L.; Valiulienè, G.; Žielienė, A.; Šimkūnaitè-Stanynienė, B.; Naruškevičius, L.; Sudavičius, A. EQCM study on the oxidation/reduction of bismuth sulfide thin films. J. Electroanal. Chem. 2010, 642, 22-29.

10. Yao, K.; Gong, W. W.; Hu, Y. F.; Liang, X. L.; Chen, Q.; Peng, L. M. Individual $\mathrm{Bi}_{2} \mathrm{~S}_{3}$ Nanowire-Based Room-Temperature $\mathrm{H}_{2}$ Sensor. J. Phys. Chem. C 20o8, 112, 8721-8724.

11. Li, H.; Yang, J.; Zhang, J.; Zhou, M. Facile synthesis of hierarchical $\mathrm{Bi}_{2} \mathrm{~S}_{3}$ nanostructures for photodetector and gas sensor. RSC Adv. 2012, 2, 6258-6261.

12. Biswas, K.; Zhao, L.-D.; Kanatzidis, M. G. Tellurium-Free Thermoelectric: The Anisotropic n-Type Semiconductor $\mathrm{Bi}_{2} \mathrm{~S}_{3}$. Adv. Energy Mater. 2012, 2, 634-638.

13. Liufu, S.-C.; Chen, L.-D.; Yao, Q.; Wang, C.-F. Assembly of one-dimensional nanorods into $\mathrm{Bi}_{2} \mathrm{~S}_{3}$ films with enhanced thermoelectric transport properties. Appl. Phys. Lett. 2007, 90, 112106.

14. Chmielowski, R.; Péré, D.; Bera, C.; Opahle, I.; Xie, W.; Jacob, S.; Capet, F.; Roussel, P.; Weidenkaff, A.; Madsen, G. K. H.; Dennler, G. Theoretical and experimental investigations of the thermoelectric properties of $\mathrm{Bi}_{2} \mathrm{~S}_{3}$. J. Appl. Phys. 2015, 117, 125103.

15. Zhang, Z.; Zhou, C.; Huang, L.; Wang, X.; Qu, Y.; Lai, Y.; Li, J. Synthesis of bismuth sulfide/reduced graphene oxide composites and their electrochemical properties for lithium ion batteries. Electrochim. Acta 2013, 114, 88-94.

16. Zhang, H.; Yang, L.; Liu, Z.; Ge, M.; Zhou, Z.; Chen, W.; Li, Q.; Liu, L. Facet-dependent activity of bismuth sulfide as lowcost counter-electrode materials for dye-sensitized solar cells. J. Mater. Chem. 2012, 22, 18572-18577.

17. Becerra, D.; Nair, M. T. S.; Nair, P. K. Analysis of a Bismuth Sulfide/Silicon Junction for Building Thin Film Solar Cells. J. Electrochem. Soc. 2011, 158, $\mathrm{H}_{741}-\mathrm{H}_{749}$.

18. Han, P.; Mihi, A.; Ferre-borrull, J.; Pallarés, J.; Marsal, L. F. Interplay Between Morphology, Optical Properties, and Electronic Structure of Solution-Processed $\mathrm{Bi}_{2} \mathrm{~S}_{3}$ Colloidal Nanocrystals. J. Phys. Chem. C 2015, 119, 10693-10699.

19. Wang, D.; Hao, C.; Zheng, W.; Ma, X.; Chu, D.; Peng, Q.; $\mathrm{Li}, \mathrm{Y} . \mathrm{Bi}_{2} \mathrm{~S}_{3}$ nanotubes: Facile synthesis and growth mechanism. Nano Res. 2009, 2, 130-134.

20. Yu, X.; Cao, C.; Zhu, H. Synthesis and photoluminescence properties of $\mathrm{Bi}_{2} \mathrm{~S}_{3}$ nanowires via surfactant micelle-template inducing reaction. Solid State Commun. 2005, 134, 239-243.

21. Liao, H.-C.; Wu, M.-C.; Jao, M.-H.; Chuang, C.-M.; Chen, Y.-F.; Su, W.-F. Synthesis, optical and photovoltaic properties of bismuth sulfide nanorods. CrystEngComm 2012, 14, 3645-3652.

22. Zhu, Y.; Murali, S.; Cai, W.; Li, X.; Suk, J. W.; Potts, J. R.; Ruoff, R. S. Graphene and Graphene Oxide: Synthesis, Properties, and Applications. Adv. Mater. 2010, 22, 3906-3924.

23. Wang, Q. H.; Kalantar-Zadeh, K.; Kis, A.; Coleman, J. N.; Strano, M. S. Electronics and optoelectronics of two-dimensional transition metal dichalcogenides. Nat. Nanotechnol. 2012, 7, 699712 .

24. Zhang, H. Ultrathin Two-Dimensional Nanomaterials. ACS Nano 2015, 9, 9451-9469.

25. Lu, Q.; Yu, Y.; Ma, Q.; Chen, B.; Zhang, H. 2D TransitionMetal-Dichalcogenide-Nanosheet-Based Composites for Photocatalytic and Electrocatalytic Hydrogen Evolution Reactions. Adv. Mater. 2016, 28, 1917-1933.

26. Mayorga-Martinez, C. C.; Sofer, Z.; Pumera, M. Layered Black Phosphorus as a Selective Vapor Sensor. Angew. Chem. Int. Ed. 2015, 127, 14525-14528.

27. $\mathrm{Bi}_{2} \mathrm{~S}_{3}$ Crystal Structure: Datasheet from "LINUS PAULING FILE Multinaries Edition - 2012" in SpringerMaterials (http://materials.springer.com/isp/crystallographic/docs/sd_19217 95), Springer-Verlag Berlin Heidelberg \& Material Phases Data System (MPDS), Switzerland \& National Institute for Materials Science (NIMS), Japan.

28. Grigas, J.; Talik, E.; Lazauskas, V. X-ray Photoelectron Spectra and Electronic Structure of $\mathrm{Bi}_{2} \mathrm{~S}_{3}$ Crystals. Phys. Status Solidi B 2002, 232, 220-230.

29. Smith, R. J.; King, P. J.; Lotya, M.; Wirtz, C.; Khan, U.; De, S.; O'Neill, A.; Duesberg, G. S.; Grunlan, J. C.; Moriarty, G.; Chen, J.; Wang, J.; Minett, A. I.; Nicolosi, V.; Coleman, J. N. Large-Scale Exfoliation of Inorganic Layered Compounds in Aqueous Surfactant Solutions. Adv. Mater. 2011, 23, 3944-3948.

3o. Lotya, M.; Hernandez, Y.; King, P. J.; Smith, R. J.; Nicolosi, V.; Karlsson, L. S.; Blighe, F. M.; De, S.; Wang, Z.; McGovern, I. T.; Duesberg, G. S.; Coleman, J. N. Liquid Phase Production of Graphene by Exfoliation of Graphite in Surfactant/Water Solutions. J. Am. Chem. Soc. 2009, 131, 3611-3620.

31. O'Neill, A.; Khan, U.; Coleman, J. N. Preparation of High Concentration Dispersions of Exfoliated $\mathrm{MoS}_{2}$ with Increased Flake Size. Chem. Mater. 2012, 24, 2414-2421.

32. Nguyen, E. P.; Carey, B. J.; Daeneke, T.; Ou, J. Z.; Latham, K.; Zhuiykov, S.; Kalantar-zadeh, K. Investigation of Two-Solvent Grinding-Assisted Liquid Phase Exfoliation of Layered $\mathrm{MoS}_{2}$. Chem. Mater. 2015, 27, 53-59.

33. Carey, B. J.; Daeneke, T.; Nguyen, E. P.; Wang, Y.; Zhen Ou, J.; Zhuiykov, S.; Kalantar-zadeh, K. Two solvent grinding sonication method for the synthesis of two-dimensional tungsten disulphide flakes. Chem. Commun. 2o15, 51, 3770-3773.

34. Coleman, J. N.; Lotya, M.; O’Neill, A.; Bergin, S. D.; King, P. J.; Khan, U.; Young, K.; Gaucher, A.; De, S.; Smith, R. J.; Shvets, I. V.; Arora, S. K.; Stanton, G.; Kim, H.-Y.; Lee, K.; Kim, G. T.; Duesberg, G. S.; Hallam, T.; Boland, J. J.; Wang, J. J.; Donegan, J. F.; Grunlan, J. C.; Moriarty, G.; Shmeliov, A.; Nicholls, R. J.; Perkins, J. M.; Grieveson, E. M.; Theuwissen, K.; McComb, D. W.; Nellist, P. D.; Nicolosi, V. Two-Dimensional Nanosheets Produced by Liquid Exfoliation of Layered Materials. Science 2011, 331, 568-571.

35. Eda, G.; Yamaguchi, H.; Voiry, D.; Fujita, T.; Chen, M.; Chhowalla, M. Photoluminescence from Chemically Exfoliated $\mathrm{MoS}_{2}$. Nano Lett. 2011, 11, 5111-5116.

36. Ambrosi, A.; Sofer, Z.; Pumera, M. Lithium Intercalation Compound Dramatically Influences the Electrochemical Properties of Exfoliated $\mathrm{MoS}_{2}$. Small 2015, 11, 605-612.

37. Ren, L.; Qi, X.; Liu, Y.; Hao, G.; Huang, Z.; Zou, X.; Yang, L.; Li, J.; Zhong, J. Large-scale production of ultrathin topological insulator bismuth telluride nanosheets by a hydrothermal intercalation and exfoliation route. J. Mater. Chem. 2012, 22, 49214926.

38. Clark, R. M.; Carey, B. J.; Daeneke, T.; Atkin, P.; Bhaskaran, M.; Latham, K.; Cole, I. S.; Kalantar-zadeh, K. Two-step synthesis of luminescent $\mathrm{MoS}_{2}-\mathrm{ZnS}$ hybrid quantum dots. Nanoscale 2015, $7,16763-16772$.

39. Zheng, J.; Zhang, H.; Dong, S.; Liu, Y.; Tai Nai, C.; Suk Shin, H.; Young Jeong, H.; Liu, B.; Ping Loh, K. High yield exfoli- 
ation of two-dimensional chalcogenides using sodium naphthalenide. Nat. Commun. 2014, 5, 2995.

40. Daeneke, T.; Clark, R. M.; Carey, B.; Ou, J. Z.; Weber, B.; Fuhrer, M. S.; Bhaskaran, M.; Kalantar-zadeh, K. Reductive exfoliation of substoichiometric $\mathrm{MoS}_{2}$ bilayers using hydrazine salts. Nanoscale 2016, 8, 15252-15261.

41. Pereira, V. M.; Castro Neto, A. H.; Liang, H. Y.; Mahadevan, L. Geometry, Mechanics, and Electronics of Singular Structures and Wrinkles in Graphene. Phys. Rev. Lett. 2010, 105, 156603.

42. Deng, S.; Berry, V. Wrinkled, rippled and crumpled graphene: an overview of formation mechanism, electronic properties, and applications. Mater. Today 2016, 19, 197-212.

43. Aresti, M.; Saba, M.; Piras, R.; Marongiu, D.; Mula, G.; Quochi, F.; Mura, A.; Cannas, C.; Mureddu, M.; Ardu, A.; Ennas, G.; Calzia, V.; Mattoni, A.; Musinu, A.; Bongiovanni, G. Colloidal $\mathrm{Bi}_{2} \mathrm{~S}_{3}$ Nanocrystals: Quantum Size Effects and Midgap States. Adv. Funct. Mater. 2014, 24, 3341-3350.

44. Salavati-Niasari, M.; Ghanbari, D.; Davar, F. Synthesis of different morphologies of bismuth sulfide nanostructures via hydrothermal process in the presence of thioglycolic acid. J. Alloys Compd. 2009, 488, 442-447.

45. Sträter, H.; ten Haaf, S.; Brüggemann, R.; Jakob, G.; Nilius, N.; Bauer, G. H. Detailed photoluminescence study of vapor deposited $\mathrm{Bi}_{2} \mathrm{~S}_{3}$ films of different surface morphology. Phys. Status Solidi B 2014, 251, 2247-2256.

46. Reshchikov, M. A.; Morkoç, H. Luminescence properties of defects in GaN. J. Appl. Phys. 2005, 97, o61301.

47. Xiong, G.; Pal, U.; Serrano, J. G. Correlations among size, defects, and photoluminescence in $\mathrm{ZnO}$ nanoparticles. J. Appl. Phys. 2007, 101, 024317.

48. Mak, K. F.; Lee, C.; Hone, J.; Shan, J.; Heinz, T. F. Atomically Thin $\mathrm{MoS}_{2}$ : A New Direct-Gap Semiconductor. Phys. Rev. Lett. 2010, 105, 136805 .

49. Zhao, Y.; Chua, K. T. E.; Gan, C. K.; Zhang, J.; Peng, B.; Peng, Z.; Xiong, Q. Phonons in $\mathrm{Bi}_{2} \mathrm{~S}_{3}$ nanostructures: Raman scattering and first-principles studies. Phys. Rev. B 2011, 84, 205330.

5o. Zumeta-Dubé, I.; Ortiz-Quiñonez, J.-L.; Díaz, D.; TralleroGiner, C.; Ruiz-Ruiz, V.-F. First Order Raman Scattering in Bulk $\mathrm{Bi}_{2} \mathrm{~S}_{3}$ and Quantum Dots: Reconsidering Controversial Interpretations. J. Phys. Chem. C 2014, 118, 30244-30252.

51. Bernechea, M.; Cao, Y.; Konstantatos, G. Size and bandgap tunability in $\mathrm{Bi}_{2} \mathrm{~S}_{3}$ colloidal nanocrystals and its effect in solution processed solar cells. J. Mater. Chem. A 2015, 3, 20642-20648.

52. Malakooti, R.; Cademartiri, L.; Akçakir, Y.; Petrov, S.; Migliori, A.; Ozin, G. A. Shape-Controlled $\mathrm{Bi}_{2} \mathrm{~S}_{3}$ Nanocrystals and Their Plasma Polymerization into Flexible Films. Adv. Mater. 2006, 18, 2189-2194.

53. Liufu, S.-C.; Chen, L.-D.; Yao, Q.; Wang, C.-F. Bismuth Sulfide Thin Films with Low Resistivity on Self-Assembled Monolayers. J. Phys. Chem. B 2006, 110, 24054-24061.

54. Moniz, S. J. A.; Blackman, C. S.; Carmalt, C. J.; Hyett, G. MOCVD of crystalline $\mathrm{Bi}_{2} \mathrm{O}_{3}$ thin films using a single-source bismuth alkoxide precursor and their use in photodegradation of water. J. Mater. Chem. 2010, 20, 7881-7886.

55. Vila, M.; Diaz-Guerra, C.; Lorenz, K.; Piqueras, J.; Alves, E.; Nappini, S.; Magnano, E. Structural and luminescence properties of $\mathrm{Eu}$ and $\mathrm{Er}$ implanted $\mathrm{Bi}_{2} \mathrm{O}_{3}$ nanowires for optoelectronic applications. J. Mater. Chem. C 2013, 1, 7920-7929.

56. Zhang, X.; Liu, Y.; Zhang, G.; Wang, Y.; Zhang, H.; Huang, F. Thermal Decomposition of Bismuth Oxysulfide from Photoelectric $\mathrm{Bi}_{2} \mathrm{O}_{2} \mathrm{~S}$ to Superconducting $\mathrm{Bi}_{4} \mathrm{O}_{4} \mathrm{~S}_{3}$. ACS Appl. Mater. Interfaces 2015, 7, 4442-4448.

57. Pacquette, A. L.; Hagiwara, H.; Ishihara, T.; Gewirth, A. A. Fabrication of an oxysulfide of bismuth $\mathrm{Bi}_{2} \mathrm{O}_{2} \mathrm{~S}$ and its photocatalytic activity in a $\mathrm{Bi}_{2} \mathrm{O}_{2} \mathrm{~S} / \mathrm{In}_{2} \mathrm{O}_{3}$ composite. J. Photochem. Photobiol., A 2014, 277, 27-36.
58. Weng, B.; Xu, F.; Xu, J. Synthesis of hierarchical $\mathrm{Bi}_{2} \mathrm{O}_{3} / \mathrm{Bi}_{4} \mathrm{Ti}_{3} \mathrm{O}_{12}$ p-n junction nanoribbons on carbon fibers from (oo1) facet dominated $\mathrm{TiO}_{2}$ nanosheets. RSC Adv. 2014, 4, 5668256689 .

59. Calzia, V.; Malloci, G.; Bongiovanni, G.; Mattoni, A. Electronic Properties and Quantum Confinement in $\mathrm{Bi}_{2} \mathrm{~S}_{3}$ RibbonLike Nanostructures. J. Phys. Chem. C 2013, 117, 21923-21929.

6o. Song, H.; Zhan, X.; Li, D.; Zhou, Y.; Yang, B.; Zeng, K.; Zhong, J.; Miao, X.; Tang, J. Rapid thermal evaporation of $\mathrm{Bi}_{2} \mathrm{~S}_{3}$ layer for thin film photovoltaics. Sol. Energy Mater. Sol. Cells 2016, 146, 1-7.

61. Chen, B.; Uher, C.; Iordanidis, L.; Kanatzidis, M. G. Transport Properties of $\mathrm{Bi}_{2} \mathrm{~S}_{3}$ and the Ternary Bismuth Sulfides $\mathrm{KBi}_{6.33} \mathrm{~S}_{10}$ and $\mathrm{K}_{2} \mathrm{Bi}_{8} \mathrm{~S}_{13}$. Chem. Mater. 1997, 9, 1655-1658.

62. Mizoguchi, H.; Hosono, H.; Ueda, N.; Kawazoe, H. Preparation and electrical properties of $\mathrm{Bi}_{2} \mathrm{~S}_{3}$ whiskers. J. Appl. Phys. 1995, 78, 1376-1378.

63. Zhao, Y.; Zhu, X.; Huang, Y.; Wang, S.; Yang, J.; Xie, Y. Synthesis, Growth Mechanism, and Work Function at Highly Oriented \{0o1\} Surfaces of Bismuth Sulfide Microbelts. J. Phys. Chem. C 2007, 111, 12145-12148.

64. Ou, J. Z.; Ge, W.; Carey, B.; Daeneke, T.; Rotbart, A.; Shan, W.; Wang, Y.; Fu, Z.; Chrimes, A. F.; Wlodarski, W.; Russo, S. P.; Li, Y. X.; Kalantar-zadeh, K. Physisorption-Based Charge Transfer in Two-Dimensional $\mathrm{SnS}_{2}$ for Selective and Reversible $\mathrm{NO}_{2}$ Gas Sensing. ACS Nano 2015, 9, 10313-10323.

65. Donarelli, M.; Prezioso, S.; Perrozzi, F.; Bisti, F.; Nardone, M.; Giancaterini, L.; Cantalini, C.; Ottaviano, L. Response to $\mathrm{NO}_{2}$ and other gases of resistive chemically exfoliated $\mathrm{MoS}_{2}$-based gas sensors. Sens. Actuators, B 2015, 207, 602-613.

66. Iqbal, N.; Afzal, A.; Cioffi, N.; Sabbatini, L.; Torsi, L. NO sensing one- and two-dimensional carbon nanostructures and nanohybrids: Progress and perspectives. Sens. Actuators, B 2013, 181, 9-21.

67. Kunakova, G.; Meija, R.; Bite, I.; Prikulis, J.; Kosmaca, J.; Varghese, J.; Holmes, J. D.; Donats, E. Sensing properties of assembled $\mathrm{Bi}_{2} \mathrm{~S}_{3}$ nanowire arrays. Phys. Scr. 2015, 90, 094017.

68. Mayorga-Martinez, C. C.; Ambrosi, A.; Eng, A. Y. S.; Sofer, Z.; Pumera, M. Metallic $1 \mathrm{~T}-\mathrm{WS}_{2}$ for Selective Impedimetric Vapor Sensing. Adv. Funct. Mater. 2015, 25, 5611-5616. 


\section{TOC Figure}

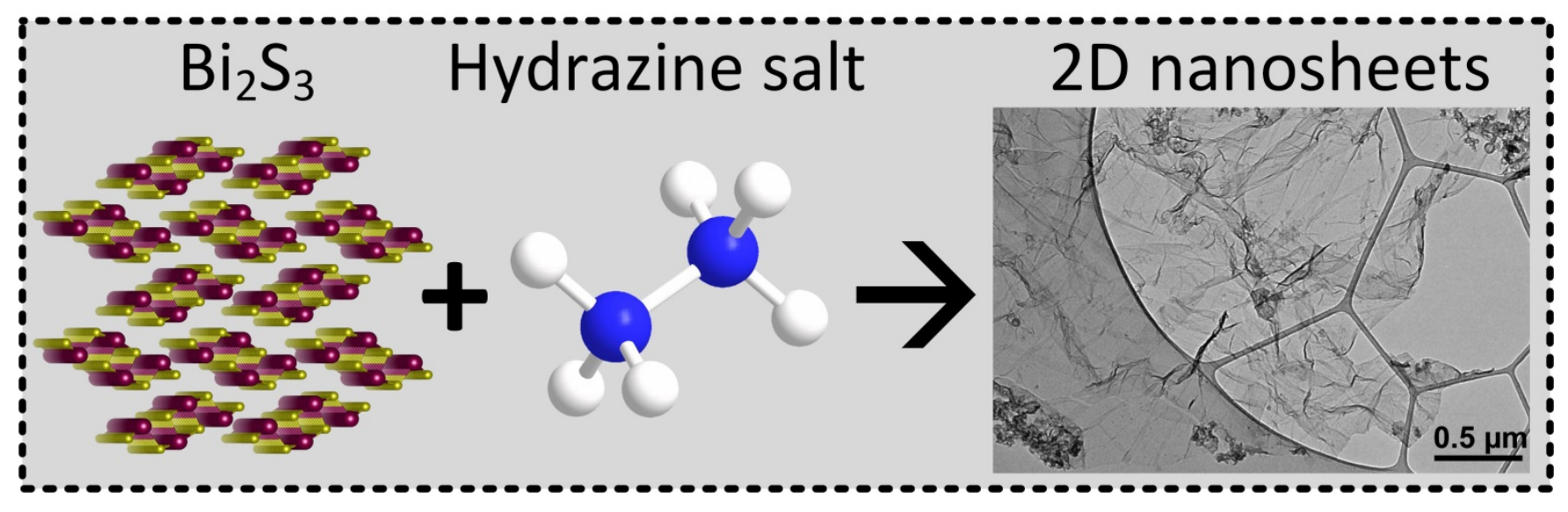

